\title{
Psicooncología
}

ISSN: $1696-7240$

\section{Evidencias psicométricas iniciales de una medida breve sobre preocu- pación por el cáncer}

Tomás Caycho-Rodríguez, ${ }^{1, *}$ José Ventura-León²; Martín Noe-Grijalva33; Miguel BarbozaPalomino ${ }^{4}$; Walter L. Arias Gallegos $;$; Mario Reyes-Bossio ${ }^{6}$; Claudio Rojas-Jara ${ }^{7}$

Resumen: Objetivo: La preocupación por el cáncer está asociada con los comportamientos preventivos para la detección de esta enfermedad; sin embargo, no existe un instrumento con evidencias de validez y confiabilidad para medir este constructo en países latinoamericanos, entre ellos el Perú. Los objetivos de este estudio fueron evaluar la validez basada en la estructura interna y fiabilidad de la Cancer Worry Scale (CWS) en una muestra de personas sin diagnóstico oncológico con historia familiar de cáncer. Método: Se contó con la participación de 215 personas sanas con historia familiar de cáncer que acudían al servicio de consulta externa de dos instituciones públicas y privadas de salud de la ciudad de Chimbote con una edad promedio de 42,10 años (DT =14,05). Para el recojo de información se utilizó una ficha sociodemográfica ad hoc y la Cancer Worry Scale. Resultados: El modelo unidimensional presentó un buen ajuste de los datos $(\mathrm{SB} \chi 2(8)=14,39$; $\mathrm{SB} \chi 2 / \mathrm{df}=1,79$; $\mathrm{CFI}=0,991$; SRMR $=$ 0,025; RMSEA = 0,061 [IC90\%: $0,000-0,111]$; AIC $=47,701)$ y una confiabilidad adecuada $(\omega$ $=0,90$; IC95\%: 0,88-0,92). Conclusión: Se concluye que la CWS presenta adecuadas propiedades psicométricas, siendo una medida que brinda interpretaciones válidas y fiables de la preocupación por el cáncer en el contexto peruano.

Palabras clave: preocupación por el cáncer; evaluación; validez; confiabilidad; Perú.

\section{[en] Initial psychometric evidence of a brief measure of cancer worry}

Abstract: Objective: The cancer worry is associated with preventive behaviors for the detection of this disease; However, there is no instrument with evidence of validity and reliability to measure this construct in Latin American countries, including Peru. The objectives of this study were to evaluate the

1 Tomás Caycho-Rodríguez. Universidad Privada del Norte, Lima, Perú

E-mail: tomas.caycho@upn.pe.

2 José Ventura-León. Universidad Privada del Norte, Lima, Perú

E-mail:jose.ventura@upn.pe

3 Martín Noe-Grijalva. Universidad César Vallejo, Chimbote, Perú

E-mail:hnoe@ucv.edu.pe

4 Miguel Barboza-Palomino. Universidad Privada del Norte, Lima, Perú

E-mail: miguel.barboza@upn.edu.pe.

$5 \quad$ Walter L. Arias Gallegos. Universidad Católica San Pablo, Arequipa, Perú

E-mail: walterlizandro@hotmail.com

6 Mario Reyes-Bossio. Universidad Peruana de Ciencias Aplicadas, Lima, Perú

E-mail: marb2383@hotmail.com

7 Claudio Rojas-Jara. Universidad Católica del Maule, Talca, Chile

E-mail: crojasj@ucm.cl

* Dirección de correspondencia: Tomás Caycho-Rodríguez, Av. Tingo María 1122, Breña, Lima. Perú.

E-mail: tomas.caycho@upn.pe. 
evidence of validity based on the internal structure and reliability of the Cancer Worry Scale (CWS) in a sample of people without cancer diagnosis with a family history of cancer. Method: It counted with the participation of 215 healthy people with a family history of cancer who attended the outpatient service of two public and private health institutions in the city of Chimbote with an average age of 42.10 years $(\mathrm{SD}=14.05)$. For the collection of information, an ad hoc sociodemographic record and the Cancer Worry Scale were used. Results: The one-dimensional model presented a good fit of the data $(\mathrm{SB} \chi 2(8)=14.39, \mathrm{SB} \chi 2 / \mathrm{df}=1.79, \mathrm{CFI}=0.991, \mathrm{SRMR}=0.025, \mathrm{RMSEA}=0.061$ [IC90\%: 0.000$0.111]$, AIC $=47.701)$ and adequate reliability $(\omega$ corrected $=0.90$; IC95\%: 0.88-0.92). Conclusion: It is concluded that the CWS presents adequate psychometric properties, being a measure that provides valid and reliable interpretations of the cáncer worry in the peruvian context.

Keywords: cancer worry; assessment; reliability; validity; Peru.

Sumario. 1. Introducción 2. Método 2.1. Participantes 2.2. Instrumentos 2.3. Procedimiento 2.4. Análisis de datos 3. Resultados 3.1. Análisis descriptivo de los ítems de la CWS 3.2. Evidencia de validez basada en el constructo y confiabilidad 4. Discusión y conclusión 5. Referencias bibliográficas Anexo 1.

Cómo citar: Caycho-Rodríguez T, Ventura-León J, Noe-Grijalva M, Barboza-Palomino M, Arias Gallegos WL, Reyes-Bossio M, Rojas-Jara C. Evidencias psicométricas iniciales de una medida breve sobre preocupación por el cáncer. Psicooncología 2018;15:315-325. doi: 10.5209/PSIC.61438.

\section{Introducción}

El cáncer es un problema de salud pública a nivel mundial, con importantes consecuencias a nivel físico, psicosocial y económico ${ }^{(1)}$. Según un informe reciente del World Cancer Report (WCR) $)^{(1)}$, en el año 2012 se registraron 14 millones de nuevos casos de pacientes con con cáncer a nivel mundial, cifra que se estima llegue a 22 millones en el 2030. El mismo informe señala que son los países de África, Asia, América Central y América del Sur donde se presentan cerca del $60 \%$ de casos diagnosticados y el 70\% de muertes por cáncer. En el Perú, entre el 2010-2012, se registró 64.243 nuevos casos de pacientes con cáncer solo en Lima, falleciendo por esta enfermedad 25.888 personas (12.540 hombres y 13.348 mujeres $)^{(2)}$. Lo anterior, hace necesario el desarrollo de mejores estrategias de detección temprana.

En este sentido, desde hace algunos años existe el interés por el estudio de las cogniciones y emociones que facilitan la presencia de comportamientos preventivos del cáncer ${ }^{(3)}$. En este contexto, se considera que la preocupación por el cáncer, definida como el conjunto de pensamientos indeseados e incontrolables acerca de la amenaza de desarrollar la enfermedad ${ }^{(4)}$, está asociada con los comportamientos preventivos para su detección ${ }^{(5)}$. La preocupación por el cáncer no solo afectaría a nivel individual sino también la vida cotidiana familiar ${ }^{(6)}$.

La literatura señala que un pequeño pero significativo porcentaje de las mujeres en la población general (12-26\%) informan tener elevados niveles de preocupación sobre el cáncer de mama ${ }^{(7)}$, mientras que entre el $1 \%$ a $3 \%$ señalan altos niveles de preocupación por el cáncer de ovario ${ }^{(8)}$. En el caso de los hombres, el 71\% informaron que no se encuentran preocupados por el cáncer de próstata ${ }^{(9)}$. Si bien la información sobre la cantidad de mujeres y hombres preocupados por el cáncer no es del todo actual, resulta aún importante para determinar el papel de la preocupación en el diagnóstico del cáncer y para entenderlo como una problemática que puede presentarse en diversos grupos de la población ${ }^{(4)}$. 
En la actualidad no esta del todo claro el vínculo entre la preocupación por el cáncer y las prácticas de detección del cáncer ${ }^{(10)}$. Por un lado, existe evidencia que sugiere que aquellas personas preocupadas por desarrollar cáncer han presentado tasas de detección más bajas ${ }^{(3)}$, mientras que otros estudios afirman que la preocupación por el cáncer genera la aparición de conductas de autoprotección y detección ${ }^{(10)}$. La contradicción en los resultados se debe en parte a los diversos métodos para medir la preocupación por el cáncer, además de la presencia de otros problemas metodológicos ${ }^{(4,10)}$. Lo anterior revela la necesidad de contar con instrumentos cuyos ítems representen adecuadamente el constructo preocupación por el cáncer y presenten adecuadas evidencias psicométricas para su medición.

La revisión de la literatura muestra la existencia de escalas breves para la medición de la preocupación por el cáncer, siendo una de las más utilizadas la Cáncer Worry Scale $(\mathrm{CWS})^{(11)}$, cuyos 6 ítems describen la frecuencia de la preocupación por el riesgo de desarrollar cáncer en un futuro y el impacto de esa preocupación en el funcionamiento diario. Si bien la CWS fue creado inicialmente para la medición de la preocupación por el cáncer de mama en el contexto estadounidense, diversos estudios han demostrado que la CWS es también útil para la medición de la preocupación por diversos tipos de cáncer y en población masculina ${ }^{(12,13)}$.

A pesar de que el estudio inicial de la CWS no reportó evidencias de validez y puntuaciones de fiabilidad, son aún pocas las investigaciones destinadas al análisis de sus propiedades psicométricas. Hopwood et al. ${ }^{(14)}$ reportaron, en una muestra de 500 mujeres con antecedentes familiares de cáncer de mama/ovario, un coeficiente alfa de cronbach para la escala igual a 0,86 demostrando una adecuada fiabilidad interna. Además, el análisis factorial mediante el método de componentes principales muestra como resultado la presencia de un solo factor que explicaba el $59,5 \%$ de la varianza. De igual manera, el estudio de la versión en español de la $\mathrm{CWS}^{(5)}$, que contó con la participación de mujeres sanas con historia familiar de cáncer de mama, indica la presencia de una estructura unifactorial que explica el $53,07 \%$ de la varianza, con un valor del coeficiente alfa de Cronbach de 0,82. Los estudios anteriores solo realizaron un análisis factorial exploratorio pero no confirmatorio. El análisis factorial confirmatorio (AFC) permite someter las hipótesis sobre la relación entre los indicadores y dimensiones latentes, a contrastes estadísticos ${ }^{(15)}$. La revisión de la literatura señala que no existe algún estudio de las propiedades psicométricas de la CWS en el contexto de países latinoamericanos, entre ellos el Perú.

En ese escenario, resulta valioso preguntarse si la CWS mostrará adecuadas propiedades psicométricas en una muestra de personas sin diagnóstico oncológico actual pero con historia familiar de cáncer. Diversos estudios señalan que las personas con antecedentes familiares de cáncer, presentan una mayor preocupación y una sobrestimación del riesgo de cáncer que se ven reflejados en mayores comportamientos preventivos $^{(4,16,17)}$. Además, estas personas, tienen un riesgo dos veces mayor, en comparación con la población general, de padecer cáncer ${ }^{(14)}$, convirtiéndose en un grupo de interés a nivel preventivo. Así, los objetivos de este estudio fueron (a) analizar de manera empírica si el modelo unidimensional original de la CWS ofrece el mejor ajuste a los datos y; (b) estimar la confiabilidad de la CWS en base al método de consistencia interna. La importancia del estudio radica en contar con una medida breve de screenning que permita identificar los diferentes niveles de preocupación por el cáncer, su relación con la aparición de comportamientos preventivos, así como mejorar la mayor comunicación centrada en el paciente y la calidad de la atención médica. 


\section{Método}

\subsection{Participantes}

El estudio instrumental contó con la participación de 215 personas sanas con historia familiar de cáncer que acudían al servicio de consulta externa de dos instituciones públicas y privadas de salud de la ciudad de Chimbote cuyas edades oscilaron entre los 20 y 88 años $(\mathrm{M}=42,10 ; \mathrm{DT}=14,05)$, donde $158(73,5 \%)$ eran mujeres y $57(26,5 \%)$ hombres. La edad promedio de mujeres $(\mathrm{M}=43,81$; DT $=14,67)$ es estadísticamente superior al promedio de edad de los hombres $(\mathrm{M}=37,37$; $\mathrm{DT}=$ $10,94)$ presentando un tamaño del efecto moderado $\left(\mathrm{t}_{(213)}=-3,02, \mathrm{p}=0,003 ; \mathrm{d}=\right.$ $0,47$; IC95\% $=0,16-0,77)$. Del total de participantes, 80 (37,2\%) son casados(as), $64(29,82 \%)$ solteros, $58(27 \%)$ convivientes y $13(6 \%)$ viudos. Los participantes infoirmaron tener un promedio de 2 familiares $(\mathrm{DT}=1,17)$ que han padecido cáncer. Además, el 14,9\% de los participantes expresaron estar muy o bastante preocupados por padecer cáncer, el $54 \%$ un poco preocupado, el 13\% nada preocupado y el $18,1 \%$ no ha pensado sobre la posibilidad de padecer cáncer.

\subsection{Instrumentos}

Ficha de datos sociodemográficos. Ficha elaborada por el equipo de investigadores que permite recoger los siguientes datos de los participantes: sexo, edad, estado civil, número de familiares que han padecido o tienen cáncer.

Cancer Worry Scale (CWS $)^{(11)}$. La CWS consta de 6 ítems destinados a medir las preocupaciones sobre desarrollar cáncer y el impacto de estas preocupaciones en el funcionamiento cotidiano de las personas en riesgo de cáncer hereditario. Se utilizó la versión en español de Cabrera et al. ${ }^{(5)}$ (Ver anexo 1). Los seis ítems presentan una escala tipo Likert de 4 puntos, donde puntuaciones elevadas expresan preocupaciones más frecuentes sobre el cáncer. La versión española reportó un coeficiente alfa de Cronbach adecuada $(\alpha=0,82)$.

\subsection{Procedimiento}

El Comité de Ética de la Universidad Privada del Norte aprobó el protocolo de investigación. Se solicitó el permiso a las autoridades de los hospitales seleccionados, informando acerca del objetivo del estudio. Obtenida la autorización se aplicó la escala de manera individual y colectiva. La totalidad de participantes recibió información sobre el objetivo del estudio y firmó el protocolo de consentimiento informado.

\subsection{Análisis de datos}

Primero, se calculó la media, desviación estándar, asimetría, curtosis. Segundo, se realizó un análisis factorial confirmatorio (AFC) utilizando el programa EQS 6.2. Se realizó un AFC debido la existencia de hipótesis previas acerca de la estructura dimensional de la CWS derivada de las soluciones factoriales reportadas en investigaciones previas. Para la estimación de los parámetros se utilizó la versión robusta del método de máxima verosimilitud (MV) y la matriz de correlación policórica, debido a la naturaleza ordinal de los ítems ${ }^{(18)}$. Se utilizó la chi-cuadrado 
corregido (SB- $\left.x^{2}\right)^{(19)}$ para evaluar la bondad de ajuste de los modelos. La SB- $x^{2}$ es sensible al tamaño muestral, por lo que se examinó la chi-cuadrada corregida relativa $\left(\mathrm{SB}-\chi^{2} / \mathrm{df}\right)$, cuyos valores $<3$ sugieren un ajuste aceptable ${ }^{(20)}$. Posteriormente, se calculó el índice de ajuste comparativo (CFI), el error cuadrático medio de aproximación (RMSEA) y el residuo cuadrático medio estandarizado (SRMR) y el criterio de información de Akaike (AIC) para la comparación de modelos. Valores de CFI $>0,95$, RMSEA y SRMR $<0,08$ expresan un buen ajuste del modelo ${ }^{(21)}$. El valor más pequeño de AIC expresa un mejor ajuste del modelo ${ }^{(22)}$. Se calculó la varianza promedio extraída del factor (AVE; Average Variance Extracted) donde un valor $\mathrm{AVE}>0,50$ permite obtener datos de validez interna discriminante ${ }^{(23)}$.

Finalmente, se estimó la confiabilidad de las variables latentes, utilizando una corrección del coeficiente omega $(\omega)$ por la presencia de errores correlacionados $\left(\omega_{\text {corregido }}\right)^{(24)}$ y sus intervalos de confianza (IC95\%) mediante el método de bootstrapping ${ }^{(25)}$. Se considera que $\omega_{\text {corregido }}>0,80$ es adecuado ${ }^{(24)}$.

\section{Resultados}

\subsection{Análisis descriptivo de los ítems de la CWS}

Los estadísticos descriptivos de los ítems de la CWS se presentan en la Tabla 1. Los ítems cuatro $(\mathrm{M}=2,39)$ y tres $(\mathrm{M}=1,83)$ presentan el mayor y menor puntaje promedio respectivamente; mientras que el ítem tres $(\mathrm{DT}=0,92)$ presenta la mayor dispersión. Los valores de asimetría y curtosis se encuentran dentro del límite esperado $(-1,5 \mathrm{a}+1,5)$.

Tabla 1. Medias, desviaciones estándar, asimetría, curtosis y correlación entre ítems de la CWS

\begin{tabular}{lcccc}
\hline Ítem & $\mathrm{M}$ & $\mathrm{DT}$ & $\mathrm{g} 1$ & $\mathrm{~g} 2$ \\
\hline Ítem 1 & 2,14 & 0,77 & 0,30 & $-0,27$ \\
Ítem 2 & 1,87 & 0,79 & 0,56 & $-0,34$ \\
Ítem 3 & 1,83 & 0,92 & 0,79 & $-0,39$ \\
Ítem 4 & 2,39 & 0,82 & 0,05 & $-0,53$ \\
Ítem 5 & 2,12 & 0,81 & 0,25 & $-0,56$ \\
Ítem 6 & 2,27 & 0,86 & $-0,02$ & $-0,86$ \\
\hline
\end{tabular}

Nota: $\mathrm{M}=$ Media; DE= Desviación Estándar; g1 = Asimetría; g2 $=$ Curtosis.

\subsection{Validez basada en el constructo y confiabilidad}

Tal como lo sugieren los autores de la traducción al español original de la $\mathrm{CWS}^{(5)}$, se probó una estructura interna unidimensional donde los seis ítems explicaban un solo factor (Modelo 1); sin embargo, las bondades del ajuste no fueron adecuadas, por lo que se re-especificó el modelo en base al índice de modificación (IM), 
observando la presencia de correlaciones entre los errores de los ítems dos y tres. De esa forma, se analizó una estructura unidimensional con la presencia de este error correlacionado (Modelo 2). Los resultados del AFC reportaron adecuados índices de ajuste para el Modelo 2 de un factor de la CWS (SB $\chi 2$ (8) =14,39; SB $\chi 2 / \mathrm{gl}=1,79$; $\mathrm{CFI}=0,991, \mathrm{SRMR}=0,025 ; \mathrm{RMSEA}=0,061[\mathrm{IC} 90 \%: 0,000-0,111]$ ) y un valor menor de AIC.

Tabla 2. Índices de bondad de ajuste estadístico de la CWS $(\mathrm{n}=215)$

\begin{tabular}{lcccccc}
\hline & $\begin{array}{c}\mathrm{SB} \chi 2 \\
(\mathrm{gl})\end{array}$ & SB $\chi 2 / \mathrm{gl}$ & SRMR & CFI & $\begin{array}{c}\text { RMSEA } \\
(\mathrm{IC} 90 \%)\end{array}$ & AIC \\
\hline $\begin{array}{l}\text { Modelo 1 } \\
\text { (original) }\end{array}$ & $\begin{array}{c}45,05 \\
(9)\end{array}$ & 5 & 0,040 & 0,950 & $\begin{array}{c}0,137 \\
(0,099, \\
0,178)\end{array}$ & 79,888 \\
\hline $\begin{array}{l}\text { Modelo 2 } \\
\text { (e2-e3) }\end{array}$ & $\begin{array}{c}\mathbf{1 4 , 3 9} \\
\mathbf{( 8 )}\end{array}$ & $\mathbf{1 , 7 9}$ & $\mathbf{0 , 0 2 5}$ & $\mathbf{0 , 9 9 1}$ & $\begin{array}{c}\mathbf{0 , 0 6 1} \\
\mathbf{( 0 , 0 0 0}\end{array}$ & $\mathbf{4 7 , 7 0 1}$ \\
\hline
\end{tabular}

Nota: $\chi^{2}=$ Chi cuadrado, $g l=$ Grados de libertad, SRMR $=$ raíz residual estandarizada cuadrática media, RMSEA = Error Cuadrático Medio de Aproximación, CFI = índice de ajuste comparativo, IC = Intervalos de confianza, $\mathrm{AIC}=$ Criterio de información de Akaike

Las cargas factoriales estandarizadas $(\lambda)$ de los ítems del Modelo 2 son significativas y en la dirección esperada, con $\lambda_{\text {promedio }}=$ de $0,802\left(\lambda_{\text {promedio }}>0,70\right)^{(26)}$. Además, se brinda evidencia de validez interna convergente $(\mathrm{AVE}=0,646)^{(23)}$. La Figura 1 muestra las cargas factoriales estandarizadas y los residuos de los ítems del Modelo 2.

Figura 1. Estructura factorial de la CWS en peruanos sanos con historia familiar de cáncer

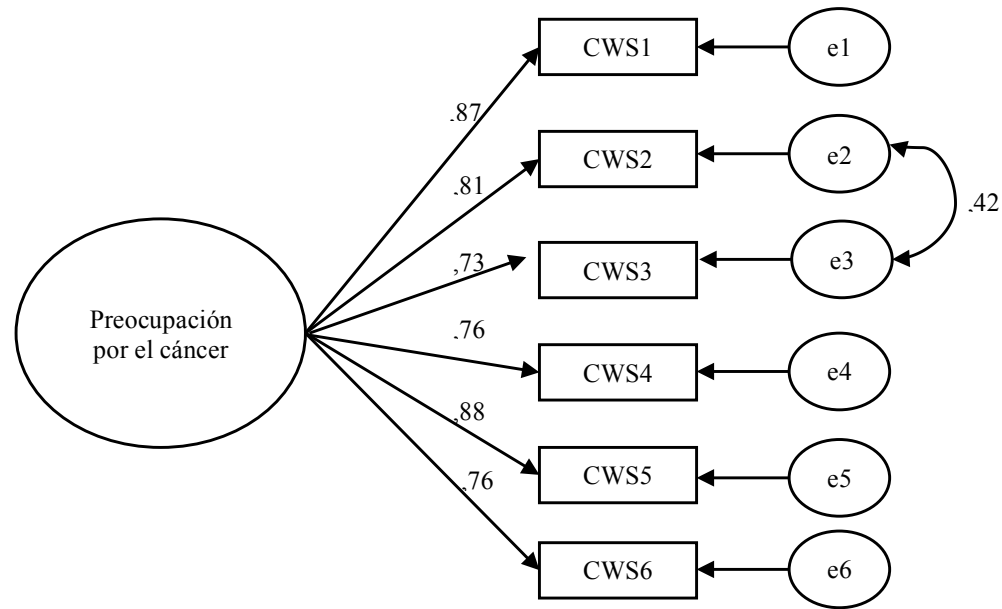

Asimismo, la confiabilidad $\left(\omega_{\text {corregido }}=0,90 ;\right.$ IC95\%: 0,88-0,92) es adecuada para el Modelo 2 unidimensional. 


\section{Discusión y conclusión}

Si bien investigaciones previas han brindado evidencia acerca la validez y confiabilidad de la $\mathrm{CWS}^{(5,14)}$, hasta donde se conoce de la literatura, este es el primer estudio publicado acerca de las evidencias psicométricas iniciales de la CWS en el Perú y Latinoamérica. En este sentido, los propósitos de este estudio fueron comprobar si el modelo unidimensional original de la CWS presenta un buen ajuste de los datos (validez basada en la estructura interna) y examinar la confiabilidad en una muestra de peruanos sanos con historial familiar de cáncer.

En primer lugar, el AFC del modelo unidimensional, basado en el estudio de la traducción al español ${ }^{(5)}$ (Modelo 1) no presentó un adecuado ajuste de los datos. El análisis de los IM sugirió que el ajuste del modelo unidimensional de la CWS podría ser satisfactorio si se correlacionan los errores de los ítems 2 y 3 . Realizados estos cambios, este segundo modelo (Modelo 2) presentó índices de ajuste aceptables. Los estudios instrumentales anteriores en España ${ }^{(5)}$ e Inglaterra ${ }^{(14)}$, no presentan errores correlacionados debido a que analizaron la estructura interna en base a un análisis factorial exploratorio (AFE); mientras que en este estudio se llevó a cabo un AFC. El $\mathrm{AFC}$, permite definir el concepto de factor común como aquel que es subyacente a los indicadores objetivos, además de no asumir igualdad de las cargas factoriales ni de las varianzas de error. Esto evitaría introducir otros factores difíciles de interpretar y someter los supuestos a contrastes estadísticos que están asociados con el análisis de la confiabilidad ${ }^{(15)}$.

En este sentido, de acuerdo a la literatura, la presencia de errores correlacionados en los modelos factoriales parece sugerir similaridad tanto en el contenido de los ítems, las demandas en las tareas, los errores de medición y el estilo de respuesta a los ítems ${ }^{(27)}$. Así, se consideró conceptualmente aceptable la presencia de errores correlacionados debido a la similaridad percibida en el contenido de ambos ítems. Tanto el ítem dos $(\diamond$ Durante el mes pasado, el pensar sobre la posibilidad de desarrollar cáncer ¿ha afectado su estado de ánimo?») y tres ( $\diamond$ Durante el mes pasado, el pensar sobre la posibilidad de desarrollar cáncer ¿ha afectado su capacidad para realizar sus actividades del día a día?») podrían percibirse como ítems relacionados a las consecuencias de la preocupación en dos procesos que se encuentran íntimamente asociados (estado de ánimo y las actividades diarias). Lo anterior se vincula con evidencia que señala que el estado de ánimo, positivo o negativo, parece ser particularmente importante para mantener la actividad diaria ${ }^{(28)}$. Estos ítems podrían revisarse, modificarse o eliminarse en futuras versiones de la CWS.

A nivel práctico, la presencia de errores correlacionados puede producir una sobrestimación del valor de los coeficientes de confiabilidad debido a la presencia de varianza no asociada con el constructo ${ }^{(29)}$ y por ende un sesgo en la interpretación de la precisión de la medición. Buscando controlar el impacto de los errores correlacionados, el análisis de la confiabilidad de las variables latentes se realizó a partir del calculó del $\omega_{\text {corregido }}$ con el objetivo de evaluar la consistencia de los ítems en la medición de la preocupación por el cáncer entre los participantes del estudio. El reporte del $\omega_{\text {corregide }}$ está más acorde con el modelo factorial utilizado y brinda una estimación más precisa de la confiabilidad ${ }^{(24)}$. El $\omega_{\text {corregido }}=0,90$ (IC95\%: 0,88-0,92) indicó que, pese a la presencia de errores correlacionados, la CWS tiene un adecuado nivel de consistencia interna para reproducir resultados similares en momentos y ocasiones diferentes $^{(24)}$ y ser útil como una medida de tamizaje. 
Los resultados contribuyen a la limitada evidencia sobre las propiedades psicométricas de las medidas sobre la preocupación por el cáncer y es de particular importancia para comprender el papel de las cogniciones y emociones asociadas con la presencia de comportamientos preventivos del cáncer ${ }^{(4)}$.

A pesar de las adecuadas propiedades psicométricas del modelo unidimensional de la CWS, se debe reconocer un conjunto de limitaciones a tomar en consideración para futuras investigaciones. En relación a los participantes del estudio, solo se recopiló información de personas adultas sanas con historia familiar de cáncer residentes en la ciudad de Chimbote. En ese sentido, futuras investigaciones deberían examinar las evidencias psicométricas de la CWS en personas de otras regiones del Perú, no solo sanas sino también en aquellas que han superado el cáncer. Esto último es importante, ya que la evidencia reporta que las personas sobrevivientes del cáncer, pueden presentar miedo constante y excesivamente elevado, que dificulta la realización de sus actividades cotidianas ${ }^{(30)}$. En segundo lugar, no se examinó la confiabilidad test-retest de la CWS, que proporcionaría evidencia empírica sobre la estabilidad del constructo preocupación por el cáncer. Tercero, solo se reportó evidencia de validez basada en el constructo, lo que hace necesario examinar otras fuentes de evidencias de validez (basada en el contenido, en la relación con otras variables, etc.).

Además, a pesar de contar con participantes varones y mujeres no se realizó un análisis de invarianza factorial (IF) debido al desigual tamaño muestral entre ambos grupos. La literatura especializada sugiere como requisito para el estudio de IF que el tamaño de las muestras fuera similar ${ }^{(31)}$. El análisis de IF es un procedimiento necesario para realizar comparaciones entre dos o más grupos (sexo, edad, estado civil, etc.) que permite tener certeza que el constructo medido (en este caso preocupación por el cáncer), tiene el mismo significado entre los grupos comparados. En este sentido, una IF no satisfactoria generaría un sesgo de medida hacia uno de los grupos, afectando la validez de las conclusiones del estudio ${ }^{(32)}$.

En conclusión, y a pesar de las limitaciones, los hallazgos aquí reportados parecen ser prometedores y sugieren que la CWS es una herramienta confiable y válida para medir la preocupación por el cáncer en el contexto peruano. Además, la rapidez y facilidad para administrar la CWS sugiere su utilidad como una medida breve de la preocupación por el cáncer en investigaciones de gran alcance.

\section{Referencias bibliográficas}

1. Stewart BW, Wild CP. World Cancer Report 2014. Lyon: International Agency for Research on Cancer, 2014.

2. Payet E, Pérez P, Poquioma E, Díaz E. Registro de cáncer de lima metropolitana. incidencia y mortalidad 2010-2012, Volumen 5. Lima: Instituto Nacional de Enfermedades Neoplásicas; 2016.

3. Bowen DJ, Helmes A, Powers D, Andersen MB, Burke W, McTiernan A, Durfy S. Predicting breast cancer screening intentions and behavior with emotion and cognition. $\mathrm{J}$ Soc Clin Psychol 2003; 22: 213-32. doi: 10.1521/jscp.22.2.213.22875

4. Hay JL, Buckley TR, Ostroff J. The role of cancer worry in cancer screening: A theoretical and empirical review of the literature. Psychooncology 2005; 14: 517-34. doi: 10.1002/ pon. 864 
5. Cabrera E, Zabalegui A, Blanco I. Spanish version of the Cancer Worry Scale (CWS). Cross cultural adaption and validity and reliability analysis. Med Clin 2011; 136: 8-12. doi: 10.1016/j.medcli.2010.04.015

6. Schnur JB, DiLorenzo TA, Montgomery GH, Erblich J, Winkel G, Hall SJ, Bovbjerg DH. Perceived risk and worry about prostate cancer: a proposed conceptual model. Behav Med 2006; 32: 89-96. doi: 10.3200/BMED.32.3.89-96

7. Sutton S, Bickler G, Sancho-Aldridge J, Saidi G. Prospective study of predictors of attendance for breast screening in inner London. J Epidemiol Commun Health 1994; 48: 65-73. doi: 10.1136/jech.48.1.65

8. Andersen MR, Peacock S, Nelson J, Wilson S, McIntosh M, Drescher C, Urban N. Worry about ovarian cancer risk and use of ovarian cancer screening by women at risk for ovarian cancer. Gynecol Oncol 2002; 85: 3-8. doi: 10.1006/gyno.2001.6556

9. Ward JE, Hughes A, Hirst GHL, Winchester L. Men's estimates of prostate cancer risk and self-reported rates of screening. Med J Aust 1997; 167: 250-3.

10. Hay JL, McCaul KD, Magnan RE. Does worry about breast cancer predict screening behaviors? A meta-analysis of the prospective evidence. Prev Med 2006: 42: 401-8. doi: 10.1016/j.ypmed.2006.03.002

11. Lerman C, Trock B, Rimer BK, Jepson C, Brody D, Boyce A. Psychological side effects of breast cancer screening. Health Psychol 1991; 10: 259.

12. Butler KM, Rayens MK, Wiggins AT, Rademacher KB, Hahn EJ. Association of smoking in the home with lung cancer worry, perceived risk, and synergistic Risk. Oncol Nurs Forum 2017; 44: E55. doi: 10.1188/17.ONF.E55-E63

13. Kelly KM, Schoenberg N, Wilson TD, Atkins E, Dickinson S, Paskett E. Cervical cancer worry and screening among Appalachian women. J Prim Prev 2015; 36: 79-92. doi: 10.1007/s10935-014-0379-7

14. Hopwood P, Shenton A, Lalloo F, Evans DG, Howell A. Risk perception and cancer worry: An exploratory study of the impact of genetic risk counselling in women with a family history of breast cancer. J Med Genet 2001; 38: 139-42. doi: 10.1136/jmg.38.2.139

15. Batista-Foguet JM, Coenders G, Alonso J. Análisis factorial confirmatorio. Su utilidad en la validación de cuestionarios relacionados con la salud. Med Clin 2004; 122: 21-7.

16. Cohen M. Breast cancer early detection, health beliefs, and cancer worries in randomly selected women with and without a family history of breast cancer. Psychooncology 2006; 15: 873-83. doi: 10.1002/pon.1018

17. Cabrera E, Blanco I, Yagüe C, Zabalegui A. The impact of genetic counseling on knowledge and emotional responses in Spanish population with family history of breast cancer. Patient Educ Couns. 2010 78: 382-8. doi: 10.1016/j.pec.2009.10.032

18. Rhemtulla M, Brosseau-Liard PÉ, Savalei V. When can categorical variables be treated as continuous? A comparison of robust continuous and categorical SEM estimation methods under suboptimal conditions. Psychol Methods 2012; 17: 354. doi: 10.1037/a0029315

19. Satorra A, Bentler PM. Corrections to test statistics and standard errors in covariance structure analysis. En: von Eye A, Clogg CC, editors. Latent variables analysis: Applications for developmental research. Thousand Oaks, CA: Sage, 1994. p.399-419

20. Schermelleh-Engel K, Moosbrugger H, Müller H. Evaluating the fit of structural equation models: Tests of significance and descriptive goodness-of-fit measures. MPR-Online 2003; 8: 23-74.

21. Hu LT, Bentler PM. Cutoff criteria for fit indexes in covariance structure analysis: conventional criteria versus new alternatives. Struct Equ Modeling 1999; 6: 1-55. doi: $10.1080 / 10705519909540118$ 
22. Burnham KP, Anderson DR. Model selection and multimodel inference: a practical information-theoretic approach. New York: Springer-Verlag, 2002.

23. Fornell C, Larcker DF. Structural equation models with unobservable variables and measurement error: Algebra and statistics. J Mark Res 1981; 382-8. doi: 10.2307/3150980

24. Raykov T. Bias of coefficient afor fixed congeneric measures with correlated errors. Appl Psychol Meas 2001; 25: 69-76. doi: 10.1177/01466216010251005

25. Ventura-León JL. Confidence Intervals for Omega Coefficient: Proposal for Calculus. Adicciones 2017; 30: 77-8. doi: 10.20882/adicciones.962.

26. Hair J, Anderson R, Tatham R, Black W. Análisis Multivariante. México, D. F.: Prentice Hall, 2014.

27. Brown T. Confirmatory factor analysis for applied research. New York: Guildford, 2006.

28. Clark LA, Watson D. Mood and the mundane: Relations between daily life events and self-reported mood. J Pers Soc Psychol 1988; 54: 296.

29. Yang Y, Green SB. A note on Structural Equation Modeling estimates of reliability. Struct Equat Modeling 2010; 17: 66-81. doi: 10.1080/10705510903438963

30. Simonelli, LE, Siegel SD, Duffy NM. Fear of cancer recurrence: A theoretical review and its relevance for clinical presentation and management. Psychooncology 2017; 26: 144454. doi: $10.1002 /$ pon. 4168

31. Byrne BM. Testing for multigroup equivalence of a measuring instrument: a walk through the process. Psicothema 2008; 20: 872-82.

32. Cheung GW, Rensvold RB. Evaluating goodness-of-fit indexes for testing measurement invariance. Struct Equat Modeling 2002; 9: 233-55. doi: 10.1207/S15328007SEM0902_5 


\section{ANEXO 1 \\ CANCER WORRY SCALE (CWS)}

Versión original de Lerman, et al. (1991)

Traducción al español de Cabrera, et al. (2011)

\section{Por favor, encierre en un círculo el número que está debajo de la respuesta más adecuada para usted:}

1. Durante el mes pasado, ¿con qué frecuencia ha pensado usted sobre sus probabilidades de desarrollar cáncer?

\begin{tabular}{|c|c|c|c|}
\hline Nunca o en raras ocasiones & Algunas Veces & A menudo & $\begin{array}{c}\text { Casi todo el } \\
\text { tiempo }\end{array}$ \\
\hline 1 & 2 & 3 & 4 \\
\hline
\end{tabular}

2. Durante el mes pasado, el pensar sobre la posibilidad de desarrollar cáncer ¿ha afectado su estado de ánimo?

\begin{tabular}{|c|c|c|c|}
\hline Nunca o en raras ocasiones & Algunas Veces & A menudo & $\begin{array}{c}\text { Casi todo el } \\
\text { tiempo }\end{array}$ \\
\hline 1 & 2 & 3 & 4 \\
\hline
\end{tabular}

3. Durante el mes pasado, el pensar sobre la posibilidad de desarrollar cáncer ¿ha afectado su capacidad para realizar sus actividades del "día a día"?

\begin{tabular}{|c|c|c|c|}
\hline Nunca o en raras ocasiones & Algunas Veces & A menudo & $\begin{array}{c}\text { Casi todo el } \\
\text { tiempo }\end{array}$ \\
\hline 1 & 2 & 3 & 4 \\
\hline
\end{tabular}

4. ¿Hasta qué punto le preocupa a usted la posibilidad de desarrollar cáncer algún día?

\begin{tabular}{|c|c|c|c|}
\hline Nada & Un poco & Bastante & Muchísimo \\
\hline 1 & 2 & 3 & 4 \\
\hline
\end{tabular}

5. ¿Con qué frecuencia se preocupa usted sobre la posibilidad de desarrollar cáncer?

\begin{tabular}{|c|c|c|c|}
\hline Nunca o en rara vez & De vez en cuando & Frecuentemente & Constantemente \\
\hline 1 & 2 & 3 & 4 \\
\hline
\end{tabular}

6. ¿El estar preocupado por desarrollar cáncer ¿es un problema importante para usted?

\begin{tabular}{|c|c|c|c|}
\hline No, en absoluto & Un poco & $\begin{array}{c}\text { Sin duda es un } \\
\text { problema }\end{array}$ & $\begin{array}{c}\text { Sí, es un proble- } \\
\text { ma muy serio }\end{array}$ \\
\hline 1 & 2 & 3 & 4 \\
\hline
\end{tabular}


